\title{
Sobrepeso e obesidade em crianças de diferentes níveis econômicos
}

\author{
Overweight and obesity in children of different \\ socioeconomic levels
}

\author{
Edna Regina Netto-Oliveira \\ Amauri Aparecido Bassoli de Oliveira ${ }^{2}$ \\ Alika Terumi Arasaki Nakashima \\ Caroline Filla Rosaneli ${ }^{2}$ \\ Albertino de Oliveira Filho ${ }^{4}$ \\ Leandro Rechenchosky ${ }^{2}$ \\ Augusto César Ferreira de Moraes ${ }^{5}$
}

1 Universidade Estadual de Maringá. Departamento de Farmácia e Farmacologia. Maringá, PR. Brasil.

2 Universidade Estadual de Maringá. Departamento de Educação Física. Maringá. PR. Brasil.

3 Pontifícia Universidade Católica do Paraná Campus Maringá. PR. Brasil.

4 Universidade Estadual de Maringá. Programa de Pós-graduação em Educação Física. Maringá. PR. Brasil.

5 Universidade de São Paulo. Programa de PósGraduação em Ciências da Faculdade de Medicina. SP. Brasil.

Recebido em 28/01/09 Revisado em 14/03/09 Aprovado em 09/06/09
Resumo - O excesso de peso está associado a inúmeros fatores, dentre eles, o nível socioeconômico das famílias. Assim, este trabalho objetivou avaliar a relação entre os níveis de sobrepeso e obesidade e a classe econômica de crianças de 6,0 a 7,9 anos, do município de Maringá/PR. O Índice de Massa Corporal (IMC) foi utilizado para diagnóstico do estado nutricional. Para a classificação econômica familiar, foi empregada proposta da Associação Brasileira de Empresas de Pesquisa (ABEP), sendo que as classes originais foram reagrupadas em níveis econômicos alto (classes A1, A2, B1), médio (classes B2, C1 e C2) e baixo (classes D e E). O tratamento estatístico foi realizado utilizando pacote computadorizado SPSS 13.0 e testes não-paramétricos e Odds Ratio (OR) com intervalo de confiança (IC) de $95 \%$. Foram avaliadas 1634 crianças de 24 escolas públicas e privadas, com idade média

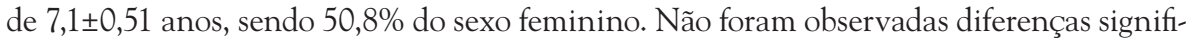
cativas entre os gêneros, para os valores de IMC, das crianças com sobrepeso e obesidade. A prevalência de excesso de peso (sobrepeso+obesidade) foi de $22,3 \%$ entre os meninos e de $21,9 \%$ entre as meninas. Não houve associação estatística entre o excesso de peso e o gênero. Por outro lado, crianças do nível econômico A apresentaram 28\% mais chance de apresentar excesso de peso que aquelas do nível B. Concluindo, neste estudo, ambos os gêneros foram igualmente afetados pelo excesso de peso, com maior prevalência entre os meninos, e as crianças pertencentes a famílias de níveis econômicos inferiores apresentaram menor chance de apresentarem excesso de peso.

Palavras-chave: Obesidade; Sobrepeso; Crianças; Nível socioeconômico.

Abstract - Excess weight is associated with various factors, including socioeconomic level. The objective of this study was to evaluate the relationship between overweight and obesity and socioeconomic level in children aged 6.0-7.9 years from Maringá, Paraná. Body mass index (BMI) was used for the assessment of nutritional status. Family socioeconomic status was classified based on the proposal of the Brazilian Association of Research Companies (ABEP), and the originally proposed classes were regrouped into high (A1, A2, and B1), medium (B2, C1, and $\mathrm{C2}$ ) and low (D and E) socioeconomic level. The data were analyzed statistically with the SPSS 13.0 program using nonparametric tests and odds ratios with a $95 \%$ confidence interval (CI). A total of 1,634 children from 24 public and private schools (mean age: $7.1 \pm 0.5$ years, $50.8 \%$ girls) were studied. No significant difference in BMI was observed between boys and girls with overweight and obesity. The prevalence of excess weight (overweight + obesity) was $22.3 \%$ and $21.9 \%$ among boys and girls, respectively. There was no significant association between excess weight and gender. However, children of high socioeconomic level had a $28 \%$ higher chance of excess weight than those of low level. In conclusion, in this study both boys and girls were equally affected by excess weight, with a higher prevalence among boys. Children from families of lower socioeconomic levels presented a lower chance of excess weight.

Key words: Obesity; Overweight; Children; Socioeconomic level. 


\section{INTRODUÇÃO}

Segundo a Organização Mundial da Saúde, a obesidade tem alcançado proporções epidêmicas globais, com mais de um bilhão de adultos com excesso de peso e, com pelo menos, 300 milhões destes clinicamente obesos. Se a prevalência continuar a aumentar na mesma proporção que nos anos 90, estima-se que 150 milhões de adultos e 15 milhões de crianças e adolescentes serão obesos, na Europa, em 2010'.

Nos últimos anos, a reversão deste quadro transformou-se numa das principais metas da Saúde Pública, particularmente, pelo fato de as populações, em idades cada vez mais precoces, serem afetadas pelo excesso de peso e suas consequências ${ }^{2-4}$.

Se para os adultos a obesidade é fator de risco para contrair um infinidade de doenças crônicas, as consequências para crianças e adolescentes são tão ou mais graves, pois eleva, precocemente, o risco para doenças cardiovasculares, problemas psicossociais, metabolismo anormal da glicose, distúrbios hepáticos e gastrintestinais, apneia do sono, complicações ortopédicas ${ }^{5}$ e distúrbios no desenvolvimento motor ${ }^{6}$. Porém, além dos indiscutíveis problemas proporcionados à saúde da criança $\mathrm{e}$ do adolescente, a obesidade em idades precoces tem como consequência persistir na idade adulta ${ }^{7}$ agravando todos os fatores de risco a ela associados ${ }^{5}$.

Segundo dados da Organização Mundial da Saúde ${ }^{8}$ 'muitas pesquisas têm destacado um aumento na prevalência de sobrepeso e obesidade entre grupos específicos, categorizados pelo fator socioeconômico. Na América Latina, indicadores da transição nutricional podem ser percebidos nos diversos países, seja na população rica, pobre, urbana ou rural. Diferentes estudos delineiam um diagnóstico semelhante, demonstrando um aumento da obesidade em todas as idades na Costa Rica, México, Guatemala, Uruguai, Chile, Honduras e Bolívia, ${ }^{910}$. Em muitos países europeus, a obesidade é mais comum entre comunidades carentes, com baixo nível salarial, educacional e reduzido acesso a cuidados básicos, o mesmo ocorrendo nos Estados Unidos ${ }^{11,12}$.

No Brasil, mudanças na política econômica do país, iniciadas há cerca de duas décadas, têm sido responsáveis por alterações demográficas, sociais e econômicas da população, as quais têm conduzido a uma situação de transição epidemiológica com substituição das doenças transmissíveis por doenças não transmissíveis e, por isso, uma situação de redução da mortalidade com aumento da morbidade da população ${ }^{13}$.
Apesar dos inegáveis benefícios sociais e de saúde proporcionados pela melhoria da renda das famílias, esta nem sempre é acompanhada por mudanças positivas, pois uma melhor situação socioeconômica tem sido associada ao maior consumo de alimentos refinados, de elevada densidade energética e de menor custo ${ }^{14}$, elevando os índices de obesidade nos países em desenvolvimento.

No Brasil, a obesidade pode ser percebida nas diferentes classes sociais e econômicas e em todas as faixas etárias e gêneros. Todavia, as últimas pesquisas em nível populacional têm demonstrado que entre as mulheres o problema vem se concentrando entre aquelas de baixo nível socioeconômico ${ }^{15}$.

Indicadores antropométricos vêm se constituindo numa forma universalmente aceita para o acompanhamento da situação nutricional e crescimento corpóreo ${ }^{16}$. A avaliação do estado nutricional na infância, por meio da aferição do peso e da estatura e, a partir deles, do cálculo do Índice de Massa Corpórea (IMC), é de grande importância, haja vista que dados antropométricos possibilitam o diagnóstico do estado nutricional de forma simples, econômica e de fácil interpretação. ${ }^{17}$

Assim, considerando a relevância do tema e o fato de ainda existirem lacunas de conhecimento em relação à epidemiologia da obesidade em nossa região e estado, este trabalho teve como objetivo avaliar a prevalência de sobrepeso e obesidade em escolares de 6,0 anos a 7,9 anos e sua relação com as diferentes classes econômicas na cidade de Maringá, Paraná, Brasil.

\section{PROCEDIMENTOS METODOLÓGICOS}

Maringá, cidade localizada no norte do estado do Paraná, possui cerca de 326.000 habitantes e, de acordo com o Atlas de Desenvolvimento Humano no Brasi $1^{18}$, apresenta Índice de Desenvolvimento Humano (IDH) de 0,841 e Índice de GINI de 0,56, com economia baseada na agricultura, comércio e prestação de serviços.

A população deste estudo, do tipo descritivo transversal ${ }^{19}$, foi composta por crianças de 6,0 anos a 7,9 anos, regularmente matriculadas em 24 escolas, sendo 17 públicas e 7 privadas, da região urbana do município de Maringá. Os dados foram coletados entre agosto e dezembro de 2006, por uma equipe de profissionais treinados, integrantes do Grupo de Estudos e Pesquisas em Obesidade e Exercício da Universidade Estadual de Maringá (GREPO/UEM).

As escolas foram selecionadas, considerando-se todos os quadrantes do Município. As crianças, da 
faixa etária em questão, de todas as escolas sorteadas, foram convidadas a participar da pesquisa, levando para casa os instrumentos de coleta de dados (Questionário da ABEP e Questionário de Dados Pessoais) a serem respondidos pelos pais ou responsáveis, bem como o Termo de Consentimento Livre e Esclarecido para assinatura. As medidas antropométricas foram efetuadas no ambiente escolar, em data previamente definida junto à escola e em local específico destinado para este fim. Foram excluídas do estudo as crianças que não retornaram o Termo de Consentimento assinado e aquelas cujas informações sobre a condição econômica, data de nascimento e/ou medidas antropométricas estavam incompletas.

As variáveis dependentes quantitativas contínuas avaliadas neste estudo foram: (a) idade, determinada com base na diferença entre a data de nascimento e a data da coleta de dados, sendo as crianças agrupadas em duas faixas etárias: 6,0-6,9 anos e 7,0-7,9 anos; (b) peso $(\mathrm{Kg})$ e, (c) estatura $(\mathrm{cm})$. As medidas de peso e estatura foram feitas em triplicata, e o valor médio calculado foi utilizado. Os equipamentos utilizados foram: balança eletrônica da marca Tanita (Modelo 2202), com capacidade para $136 \mathrm{~kg}$ e precisão de $100 \mathrm{~g}$ e um estadiômetro da marca SECA (Modelo Bodymeter 206). Os níveis de sobrepeso e a obesidade foram definidos a partir do Índice de Massa Corporal (IMC), com base nos pontos de corte ajustados para gênero e idade propostos por Cole et $\mathrm{al}^{20}$. Também foi considerado desfecho para o estudo o excesso de peso, obtido a partir do agrupamento das crianças com sobrepeso e obesidade, particularmente, quando empregadas as medidas de efeito e associação.

As variáveis independentes consideradas foram gênero e nível econômico. Este índice considerado segundo o Critério de Classificação Econômica Brasil, da Associação Brasileira de Empresas de Pesquisa $(\mathrm{ABEP})^{21}$. Para fins de análise, os oito níveis econômicos, ou classes econômicas, previstos pela ABEP, foram reagrupados e nominados da seguinte forma: A1, A2 e B1 em Nível Econômico Alto (A); B2, C1 e C2 em Nível Econômico Médio (M) e, D e E em Nível Econômico Baixo (B).

O tratamento estatístico foi realizado mediante o pacote computadorizado SPSS 13.0. As possíveis diferenças entre os gêneros para as variáveis quantitativas foram verificadas por meio do Teste U de MannWhitney. A análise do IMC por classe econômica foi feita por Kruskal Wallis e as diferenças entre as classes pelo Teste U de Mann-Whitney. Utilizou-se Odds Ratio (OR) como medida de efeito, com intervalo de confiança de 95\% (IC95\%), para analisar a associação entre o excesso de peso e as variáveis independentes. Na análise de regessão, o modelo foi ajustado para o nivel socioeconômico, por considerarmos uma variável de confundimento. Utilizaram-se os testes de Wald para heterogeneidade para variáveis dicotômicas ou nominais e de tendência linear para variáveis categóricas ordinais. O critério de significância estatística estabelecido foi de 5\%.

A pesquisa foi conduzida dentro dos padrões exigidos pela Declaração de Helsinque e aprovada pela comissão de ética da Universidade Estadual de Maringá (UEM) (Parecer n. 016/2006), tendo sido garantido o sigilo individual dos dados.

\section{RESULTADOS}

A idade média das 1.634 crianças avaliadas foi de $7,1 \pm 0,51$ anos e a amostra foi composta por $50,8 \%$

Tabela 1. Distribuição do estado nutricional de acordo com o gênero das crianças de 6,0-7,9 anos de escolas da zona urbana de Maringá/PR, 2006.

\begin{tabular}{|c|c|c|c|c|c|c|c|}
\hline $\begin{array}{l}\text { Estado } \\
\text { nutricional }\end{array}$ & \multicolumn{2}{|c|}{ Masculino } & \multicolumn{2}{|c|}{ Feminino } & $\mathrm{p}$ & \multicolumn{2}{|c|}{ Total } \\
\hline Eutrofia & $38,2(625)$ & $\begin{array}{c}15,7 \pm 1,24 \\
(10,1 \sim 18,4)\end{array}$ & $\begin{array}{l}39,7 \\
(648)\end{array}$ & $\begin{array}{l}15,4 \pm 1,19 \\
(9,3 \sim 18,3)\end{array}$ & $0,001^{a, b}$ & 77,9 (1273) & $\begin{array}{l}15,6 \pm 1,22 \\
(9,3 \sim 18,4)\end{array}$ \\
\hline Obesidade & $4,3(70)$ & $\begin{array}{c}23,2 \pm 2,52 \\
(20,3 \sim 34,4)\end{array}$ & $\begin{array}{l}4,0 \\
(65)\end{array}$ & $\begin{array}{c}23,0 \pm 1,98 \\
(20,3 \sim 28,9)\end{array}$ & $0,919^{\underline{a}}$ & 8,3 (135) & $\begin{array}{c}23,1 \pm 2,27 \\
(20,3 \sim 34,4)\end{array}$ \\
\hline $\begin{array}{l}\text { Excesso de peso } \\
(\mathrm{S}+\mathrm{O})\end{array}$ & $11,0(179)$ & $\begin{array}{c}20,7 \pm 2,61 \\
(17,6 \sim 34,4)\end{array}$ & $11,1(182)$ & $\begin{array}{c}20,5 \pm 2,33 \\
(17,3 \sim 28,9)\end{array}$ & $0,511^{a}$ & $22,1(361)$ & $\begin{array}{l}20,6 \pm 2,47 \\
(17,3 \sim 34,4)\end{array}$ \\
\hline Total & $49,2(804)$ & $16,8 \pm 2,66$ & $50,8(830)$ & $16,5 \pm 2,57$ & $0,012^{a, b}$ & $100,0(1634)$ & $\begin{array}{l}16,7 \pm 2,61 \\
(9,3 \sim 34,4)\end{array}$ \\
\hline
\end{tabular}

a Teste "U" de Mann-Whitney

${ }^{\mathrm{b}} \mathrm{p}<0,05$ (diferença significativa entre os gêneros) 
$(n=830)$ de crianças do sexo feminino. Os valores de IMC não diferiram de forma significativa entre as faixas etárias estudadas, sendo em média de 16,6 2,60 $\mathrm{kg} / \mathrm{m}^{2}$ para as crianças de 6,0-6,9 anos $(\mathrm{n}=494)$ e de $16,7 \pm 2,66 \mathrm{~kg} / \mathrm{m}^{2}$ para as de 7,0-7,9 anos $(\mathrm{n}=1140)$.

A classificação do estado nutricional com base no IMC indicou que 22,1\% das crianças se encontravam com excesso de peso, sendo $13,8 \%$ com sobrepeso e 8,3\% com obesidade (Tabela 1).

Ainda, conforme apresentado na Tabela 1, quando avaliado por gênero, o IMC médio dos meninos foi significativamente superior ao das meninas, respectivamente, $16,8 \pm 2,66 \mathrm{~kg} / \mathrm{m}^{2}$ e $16,5 \pm 2,57$ $\mathrm{kg} / \mathrm{m}^{2}$, todavia não foram observadas diferenças significativas entre os IMCs médios de meninos e meninas com excesso de peso, sobrepesados e obesos. Quando o estado nutricional foi avaliado para cada um dos sexos, separadamente (valores não apresentados na tabela), verificou-se que entre os meninos 22,3\% apresentaram excesso de peso, enquanto entre as meninas $21,9 \%$ se encontravam nesta categoria. Muito embora a prevalência de excesso de peso tenha sido muito próxima, entre as meninas houve maior prevalência de sobrepeso em relação aos meninos (14,1\% e 13,6\%, respectivamente), enquanto entre os meninos a prevalência de obesidade foi superior a verificada entre as meninas (8,7\% e 7,8\%, respectivamente).

De acordo com o critério de classificação econômica empregado neste estudo, mais de dois terços $(69,6 \%)$ das crianças avaliadas pertenciam ao nível
M, enquanto $23,4 \%$ pertenciam a famílias de nível $\mathrm{A}$ e $7,0 \%$ às de nível $\mathrm{B}$.

A análise dos resultados (Tabela 2) indicou que os valores de IMC diferiram entre os níveis econômicos ( $\mathrm{p}=0,002)$. Quando considerados os IMCs médios totais por nível econômico, as crianças do nível A apresentaram IMC significativamente superior $\left(17,1 \mathrm{Kg} / \mathrm{m}^{2}\right)$ àquelas dos níveis $\mathrm{B}\left(16,6 \mathrm{Kg} / \mathrm{m}^{2}\right) \mathrm{e}$ $\mathrm{C}\left(16,2 \mathrm{Kg} / \mathrm{m}^{2}\right)$. Quando considerados os IMCs das crianças com sobrepeso, estes não diferiram entre os três níveis considerados, o mesmo ocorrendo entre as crianças obesas. Por outro lado, quando considerado o desfecho excesso de peso, as crianças do nível A apresentaram o menor valor de IMC.

Considerando-se as prevalências dos diferentes estados nutricionais, nos diferentes níveis econômicos estudados, verificou-se que entre as crianças de nível econômico superior, a prevalência de excesso de peso foi nítidamente superior, sendo de 28,5\%, $20,5 \%$ e $16,7 \%$ para os níveis A, B e C, respectivamente (Tabela 2).

Quando avaliadas as chances de uma criança apresentar excesso de peso (Odds ratio), verificouse que não houve associação estatística entre o excesso de peso e o gênero da criança (Tabela 3), ou seja, na amostra analisada, meninos e meninas têm a mesma chance de apresentar excesso de peso, apesar de a prevalência ter sido maior entre os meninos (22,3\%) em relação às meninas (21,9\%). Por outro lado, o excesso de peso associou-se com o nível econômico de forma distinta: positivamente

Tabela 2. Distribuição do estado nutricional de crianças de 6,0 a 7,9 anos de Maringá/PR de acordo com o nível econômico, 2006.

\begin{tabular}{|c|c|c|c|c|c|c|}
\hline \multirow{3}{*}{$\begin{array}{l}\text { Estado } \\
\text { Nutricional }\end{array}$} & \multicolumn{6}{|c|}{ Nível econômico } \\
\hline & \multicolumn{2}{|c|}{ Baixo (B) } & \multicolumn{2}{|c|}{ Médio (M) } & \multicolumn{2}{|c|}{ Alto (A) } \\
\hline & $\%(N)$ & $\begin{array}{c}\mathrm{IMC}\left(\mathrm{kg} / \mathrm{m}^{2}\right) \\
\text { média } \pm \mathrm{dp} \\
(\min \sim \max )\end{array}$ & $\%(\mathrm{~N})$ & $\begin{array}{l}\mathrm{IMC}\left(\mathrm{kg} / \mathrm{m}^{2}\right) \\
\text { média } \pm \mathrm{dp} \\
(\min \sim \max )\end{array}$ & $\%(\mathrm{~N})$ & $\begin{array}{l}\text { IMC }\left(\mathrm{kg} / \mathrm{m}^{2}\right) \\
\text { média } \pm \mathrm{dp} \\
(\min \sim \max )\end{array}$ \\
\hline Eutrofia & $\begin{array}{l}83,3 \\
(95)\end{array}$ & $\begin{array}{c}15,3^{\mathrm{a}} \\
\pm 1,25 \\
(12,0 \sim 17,6)\end{array}$ & $\begin{array}{l}79,5 \\
(905)\end{array}$ & $\begin{array}{c}15,5^{\mathrm{a}} \\
\pm 1,21 \\
(9,3 \sim 18,4)\end{array}$ & $71,5(273)$ & $\begin{array}{c}15,8^{\mathrm{b}} \\
\pm 1,20 \\
(12,5 \sim 18,4)\end{array}$ \\
\hline Sobrepeso & $\begin{array}{l}11,4 \\
(13)\end{array}$ & $\begin{array}{c}19,1^{\mathrm{a}} \\
\pm 0,81 \\
(18,2 \sim 20,6)\end{array}$ & $\begin{array}{l}12,5 \\
(142)\end{array}$ & $\begin{array}{c}19,2^{\mathrm{a}} \\
\pm 0,91 \\
(17,3 \sim 21,4)\end{array}$ & $\begin{array}{l}18,6 \\
(71)\end{array}$ & $\begin{array}{c}18,9^{\mathrm{a}} \\
\pm 0,74 \\
(17,4 \sim 21,0)\end{array}$ \\
\hline Obesidade & $\begin{array}{l}5,3 \\
(6)\end{array}$ & $\begin{array}{c}23,7^{\mathrm{a}} \\
\pm 2,09 \\
(21,5 \sim 26,1)\end{array}$ & $\begin{array}{l}8,0 \\
(91)\end{array}$ & $\begin{array}{c}23,2^{\mathrm{a}} \\
\pm 2,16 \\
(20,3 \sim 30,3)\end{array}$ & $\begin{array}{l}9,9 \\
(38)\end{array}$ & $\begin{array}{c}22,7^{\mathrm{a}} \\
\pm 2,53 \\
(20,3 \sim 34,4)\end{array}$ \\
\hline $\begin{array}{l}\text { Excesso de } \\
\text { peso }(\mathrm{S}+\mathrm{O})\end{array}$ & $\begin{array}{l}16,7 \\
(19)\end{array}$ & $\begin{array}{c}20,5^{\mathrm{a}, \mathrm{b}} \\
\pm 2,57 \\
(18,2 \sim 26,1)\end{array}$ & $\begin{array}{l}20,5 \\
(233)\end{array}$ & $\begin{array}{c}20,7^{\mathrm{a}} \\
\pm 2,48 \\
(17,3 \sim 30,3)\end{array}$ & $\begin{array}{l}28,5 \\
(109)\end{array}$ & $\begin{array}{c}20,2^{\mathrm{b}} \\
\pm 2,41 \\
(17,4 \sim 34,4)\end{array}$ \\
\hline Total & $\begin{array}{l}100 \\
(114)\end{array}$ & $\begin{array}{c}16,2^{\mathrm{a}} \\
\pm 2,49 \\
(12,0 \sim 26,1)\end{array}$ & $\begin{array}{l}100 \\
(1138)\end{array}$ & $\begin{array}{c}16,6^{\mathrm{a}} \\
\pm 2,63 \\
(9,3 \sim 30,3)\end{array}$ & $\begin{array}{l}100 \\
(382)\end{array}$ & $\begin{array}{c}17,1^{\mathrm{b}} \\
\pm 2,56 \\
(12,5 \sim 34,4)\end{array}$ \\
\hline
\end{tabular}

${ }^{a}$ Kruskal Wallis e teste Post Hoc de Mann-Whitney. Diferença significativa para $\mathrm{p}<0,05$.

b,cLetras iguais numa mesma linha indicam não haver diferença significativa entre as médias de IMC dos níveis econômicos 
para as crianças do nível econômico $A$, quando comparadas com as do nível B (OR: 1,28, IC: 1,06-1,54), com forte associação dose-resposta e, negativamente, para as do nível $\mathrm{M}$ média, quando comparadas com as da classe baixa (OR: 0,82, IC: 0,7-0,96). Assim, neste estudo, crianças do nível A apresentaram 28\% mais chance de ter excesso de peso que aquelas do nível $\mathrm{B}$ e, pertencer ao nível econômico $\mathrm{M}$ protege as crianças contra o excesso de peso, em comparação com as do nível $\mathrm{B}$, indicando que apresentam menor probabilidade de ter excesso de peso.

\section{DISCUSSÃO}

A prevalência de excesso de peso verificada neste estudo $(22,1 \%)$ foi inferior à encontrada por Guedes et $\mathrm{al}^{22}$ que, ao investigarem escolares de Londrina/ PR, encontraram no grupo etário de 7-10 anos, 24,5\% de crianças nessa condição. Todavia, o percentual de crianças obesas aqui obtido foi duas vezes superior ao verificado por aqueles autores $(4,3 \%)$.

Por outro lado, essa prevalência foi superior àquela observada por Abrantes et $\mathrm{al}^{23}$,em crianças menores de 10,9 anos das regiões Nordeste e Sudeste do Brasil. Os autores encontraram excesso de peso em 18,1\% da amostra, sendo 10,8\% de sobrepesados e de 7,3\% de obesos. Os valores aqui observados também foram superiores aos encontrados em escolares de 6-10 anos na cidade de Brasília, entre os quais, $14,6 \%$ das crianças apresentaram sobrepeso e 5,5\% obesidade ${ }^{24}$. De acordo com estudos descritos por Araújo e Petroski ${ }^{25}$, entre escolares da região sul do Brasil, são constatados índices de obesidade e sobrepeso superiores aos das regiões Sudeste, Nordeste, Norte e Centro-Oeste, isto porque, em função da transição nutricional vigente, os índices de obesidade são crescentes nas regiões mais ricas do país.
Num estudo realizado no interior do Paraná, em 2002-2003, com meninos e meninas de origem germânica e não germânica, utilizando IMC como referência, 8,8\% dos meninos e 6,6\% das meninas de origem germânica se encontravam obesos e entre os que não tinham esta origem étnica, 10,9\% estavam obesos ${ }^{26}$.

O crescente aumento da prevalência de sobrepeso e obesidade entre crianças e jovens que vem sendo reportado em trabalhos realizados nas diferentes regiões do país, constitui-se num importante sinal de alerta para as autoridades de saúde, uma vez que IMCs acima dos valores normais estão associados ao desenvolvimento de várias patologias, já em idades precoces, dentre elas, níveis inadequados de pressão arteriall $^{27}$.

Os resultados aqui apresentados indicam que, embora as crianças de nível econômico A com excesso de peso tenham apresentado o menor IMC médio, a prevalência de crianças nesta condição foi muito superior à verificada nos níveis $\mathrm{M}$ e $\mathrm{B}$. Os resultados também demonstram que o problema do excesso de peso permeia todas as classes econômicas, evidenciando perfeitamente a fase de transição nutricional pela qual as sociedades em desenvolvimento, como o Brasil, estão passando, a saber, a prevalência simultânea de excesso de peso entre as camadas mais ricas e mais pobres da sociedade.

Os resultados de estudos nos quais o estado nutricional foi avaliado, segundo duas classes econômicas (alta e baixa), indicaram uma prevalência de excesso de peso significativamente superior entre as crianças brasileiras de famílias economicamente mais favorecidas em relação às de menor renda ${ }^{23,28}$. Mesmo para grupos com idades entre 10 e 19 anos, o excesso de peso continuou sendo maior entre os adolescentes de famílias das classes econômicas $A$ e B, do que nas das classes C, D e $E^{29}$.

Tabela 3. Análise bruta e ajustada associação entre o excesso de peso e o gênero da criança

\begin{tabular}{|c|c|c|c|c|}
\hline \multirow[b]{2}{*}{ Variáveis } & \multicolumn{2}{|c|}{ Análise bruta } & \multicolumn{2}{|c|}{ Análise ajustada } \\
\hline & $\mathrm{RO}(\mathrm{IC} 95 \%)$ & $\mathrm{p}$ & $\mathrm{RO}(\mathrm{IC} 95 \%)$ & $p$ \\
\hline Sexo & & $0,84^{*}$ & & $0,42^{*}$ \\
\hline Feminino & 1 & & 1 & \\
\hline Nível econômico & & $0,23^{* *}$ & & $0,01^{* *}$ \\
\hline Médio (M) & $1,28(0,77-2,15)$ & & $0,82(0,70-0,96)$ & \\
\hline Baixo (B) & 1 & & 1 & \\
\hline
\end{tabular}


Conforme Adjemian et $\mathrm{al}^{9}$, a relação entre nível socioeconômico e aumento de peso é um tema de relevância atual, pois auxilia no estabelecimento do perfil dos grupos de risco para aumento de peso em vários países da América Latina. Em estudo de nível populacional realizado na cidade de Santos, São Paulo, com crianças de 7-10 anos, a prevalência do excesso de peso entre as crianças foi de 33,7\%, bem superior àquela aqui apresentada, sendo superior entre as crianças oriundas de escolas privadas e, portanto, de maior renda ${ }^{30}$.

Os resultados obtidos que indicaram a maior chance de uma criança de nível econômico alto apresentar excesso de peso corroboram aqueles obtidos por Guedes et $\mathrm{al}^{22}$, cujas estimativas encontradas revelaram que as prevalências associadas ao sobrepeso e à obesidade apresentaram valores progressivamente maiores de acordo com posições econômicas mais elevadas. Esses autores verificaram que escolares de nível econômico mais privilegiado demonstraram risco, aproximadamente, duas vezes maior de apresentar excesso de peso em relação aos de nível mais baixo.

Evidências como estas apontam a necessidade de medidas de intervenção, particularmente, em nível escolar e de saúde pública, como alternativa para minimizar o problema da obesidade em nossa sociedade.

A principal limitação verificada acreditamos ter sido aquela presente em todos os estudos que envolvem a coleta de dados fornecidos por terceiros, aqui neste caso, pais ou responsáveis pelas crianças. Há que se supor como verdadeiras todas as informações referentes ao preenchimento do questionário usado para a classificação econômica. Todavia, por se tratar de um instrumento simples, acreditamos que a provável margem de erro tenha sido pequena e, por isso, os resultados obtidos não perdem sua grande relevância em subsidiar ações de autoridades em todas as esferas governamentais, haja vista o caráter epidemiológico do estudo.

\section{CONCLUSÃO}

Neste estudo, observou-se que meninos e meninas, na faixa etária avaliada, são indistintamente atingidos pelo excesso de peso e que aqueles pertencentes às famílias de níveis econômicos superiores, apresentaram maior chance de manifestar o problema.

Mudanças comportamentais e no estilo de vida são necessárias para o combate desse grave problema de saúde pública no qual se transformou a obesidade no Brasil e no mundo. Assim, o monitoramento do ganho de peso, do consumo e do gasto energético tornam-se de real importância para a promoção da saúde. Mais ainda, é necessário que os hábitos, informações ou experiências transmitidos e ensinados se incorporem no dia-a-dia das crianças, passando a ser adotados a posteriori do período de escolarização, por toda a vida.

Estratégias integradas de aproximação com o sistema educacional, envolvendo, além dos professores e funcionários das escolas, a participação da família e de profissionais de saúde, tais como a realização e cursos de atualização e aperfeiçoamento de professores, cursos e palestras sobre alimentação saudável e outros, são fundamentais na expectativa de que fomentem a adoção de hábitos de vida mais saudáveis e promovam as mudanças individuais e coletivas necessárias. $\mathrm{O}$ ambiente escolar propicia oportunidades de atuação, bem como o envolvimento de alunos, familiares e educadores em ações conjuntas que, espera-se, sejam eficazes na prevenção do excesso de peso na infância. No entanto, para que o processo de mudança seja efetivo, pressupõe-se a atuação de professores minimamente capacitados nas questões de saúde pública, pois só assim poderão se tornar agentes transformadores de saúde para seus alunos.

\section{REFERÊNCIAS BIBLIOGRÁFICAS}

1. WHO. World Health Organization. The challenge of obesity in the WHO European Region. Copenhagen, Bucharest; 2005.

2. Taveras EM, Berkey CS, Rifas-Shiman SL, Ludwig DS, Rockett HRH, Field AE, et al. Association of consumption of fried food away from home with body mass index and diet quality in older children and adolescents. Pediatrics 2005;116(4):e518-e524.

3. Neville L, Thomas M, Bauman A. Food advertising on Australian television - the extent of childrens exposure. Health Promot Int Oxford 2005;20(2):105-112.

4. Monteiro CA, Moura EC, Conde WL, Popkin BM. Socioeconomic status and obesity in adult populations of developing countries: a review. Bull World Health Organ 2004;82(12):940-946.

5. OMS. Organização Mundial da Saúde. Obesidade: prevenindo e controlando a epidemia global. Relatório da consultoria da OMS, São Paulo: Roca; 2004.

6. Pazin J, Frainer DES. Obesity and motor development: a cross-sectional study with Brazilians school children. FIEP Bulletin 2007;77(SE):453-456.

7. Braet C, Tanghe A, Decaluwé V, Moens E, Rosseel Y. Impatient treatement for children with obesity: weight loss, phychological well-being, and eating behavior. J Pediatr Psycol 2004;29(7):519-529. 
8. WHO. World Health Organization. The challenge of obesity in the WHO European Region and the strategies for response: summary. Branca F, Nikogosian $\mathrm{H}$, Lobstein T, eds. Dinamarca, 2007.

9. Adjemian D, Bustos P, Amigo H. Nivel socioeconómico y estado nutricional: un estudio en escolares. Arch Latinoam Nutr 2007;57(2):125-129.

10. Uauy R, Albala C, Kain J. Obesity trends in Latin American: transiting from under to overweight. J Nutr 2001;131(3):893-899.

11. Cossrow N, Falkner B. Race/ethnic issues in obesity and obesity-related co-morbidities. The J Clin Endoc Met 2004;89(6):2590-2594.

12. Crawford D, Ball K. Behavioral determinants of the obesity epidemic. Asia Pacific J Clin Nut 2002;11(SE):S718-S721.

13. Schramm JMA, Oliveira AF, Leite IC, Valente JG, Gadelha AMJ, Portela MC, Campo MR. Transição epidemiológica e o estudo de carga de doença no Brasil. Ciênc Saúde Coletiva 2004;9(4): 897-908.

14. Drewnowski A, Darmon N. The economics of obesity: dietary energy density and coats. Am J Clin Nutr 2005;82(1S):265S-273S.

15. Ferreira VA, Magalhães R. Obesidade e pobreza: o aparente paradoxo. Um estudo com mulheres da Favela da Ricinha, Rio de Janeiro, Brasil. Cad Saúde Pública 2005;21(6):1792-1800.

16. Salvo VLMA, Gimeno SGA. Reprodutibilidade e valiadade do questionário de freqüência de consumo de alimentos. Rev Saúde Publica 2002;36(4):505-12.

17. Salomons E, Rech CR, Loch MR. Estado nutricional de escolares de seis a dez anos de idade da rede municipal de ensino de Arapoti, Paraná. Rev Bras Cineantropom Desempenho Hum 2007;9(3):244-249.

18. PNUD. Programa das Nações Unidas para o Desenvolvimento. Atlas do Desenvolvimento Humano. Disponível: http://www.pnud.org.br/atlas . Acesso em 26 nov 2007.

19. Medronho RA. Epidemiologia: História e Fundamentos. Atheneu: Sao Paulo. 2006.

20. Cole TJ, Bellizzi MC, Flegal KM, Dietz WH. Establishing a standard definition for child overweight and obesity worldwide: international survey. Br Med J 2000;320:1240-1243.

21. ABEP. Associação Brasileira de Empresa e Pesquisa. CCEB. Critério de Classificação Econômica Brasil, 2008. Disponível em http://www.abep.org.br . Acesso em 10 mar 2008.
22. Guedes DP, Paula IG, Guedes JERP, Stanganelli LCR. Prevalência de sobrepeso e obesidade em crianças e adolescentes: estimativas relacionadas ao sexo, à idade e à classe socioeconômica. Rev Bras Educ Fís Esp 2006;20(3):151-163.

23. Abrantes MM, Lamounier JA, Colosimo EA. Prevalência de sobrepeso e obesidade nas regiões nordeste e sudeste do Brasil. Rev Assoc Med Bras 2003;49(2):162-166.

24. Giugliano R, Melo ALP. Diagnóstico de sobrepeso e obesidade em escolares: utilização do índice de massa corporal segundo padrão internacional. J Pediatr (Rio J) 2004;80(2):129-134.

25. Araújo EDS, Petroski EL. Estado nutricional e adiposidade de escolares de diferentes cidades brasileiras. Rev Educação Física/UEM 2002;13(2):47-53.

26. Roman EP, Barros Filho AA. Diferenças no crescimento e na composição corporal entre escolares de origem germânica e brasileira. Rev Pau. Pediatr 2007;25(3)227-232.

27. Monego E T, Jardim PCBV. Determinantes de risco para doenças cardiovasculares em escolares. Arq Bras Cardiol 2006;87(1):37-45.

28. Batista Filho M, Rissin A. A transição nutricional no Brasil: tendências regionais e temporais. Cad Saúde Públ 2003;19(supl 1):S181-S191.

29. Nunes MMA, Figueiroa JN, Alves JGB. Excesso de peso, atividade física e hábitos alimentares entre adolescentes de diferentes classes econômicas em Campina Grande (PB). Rev Assoc Med Bras 2007;53(2):130-134.

30. Costa RF, Cintra IP, Fisberg M. Prevalência de sobrepeso e obesidade em escolares da cidade de Santos, SP. Arq Bras Endocr Metab 2006;50(1):60-67.

\section{Agradecimentos}

Conselho Nacional de Desenvolvimento Científico e Tecnológico (CNPq), Processo n 402162/2005-6.

\section{Endereço para correspondência}

Edna Regina Netto de Oliveira.

Universidade Estadual de Maringá - Departamento de Farmácia e Farmacologia/CCS.

Av. Colombo, n 5790, Campus Universitário, CEP: 87020-900. Maringá, PR, Brasil.

E-mail: ernoliveira@uem.br 